\title{
Mechanism Modeling and Simulation of Coagulation Sedimentation Process
}

\author{
Wenxuan Zhao ${ }^{1}$, Xiaorong $\mathrm{Hu}^{1}$, Wenbing $\mathrm{Zhu}^{1}$ and Yunlong $\mathrm{Liu}^{1, *}$ \\ ${ }^{1}$ Department of Automation, Xiamen University, Xiamen, China \\ *corresponding author: ylliu@xmu.edu.cn
}

\begin{abstract}
Keywords: Coagulation sedimentation process; Mechanism modeling; Coagulant dosage control; Population balances.
\end{abstract}

\begin{abstract}
In this paper, we analyzed the mechanism of coagulation sedimentation process in water treatment and proposed a mechanism model according to the population balances and the characteristics of the system. The model consists of mixing, flocculation and sedimentation, and reflects the relationship of original water turbidity, coagulant dosage and the water turbidity after sedimentation. Furthermore, we simulated and analyzed the effects of mutations in original water turbidity with different initial values and different coagulant dosages on the water turbidity after sedimentation. The studies show that the model can be used for the study of coagulant dosage control, and contribute to a better understanding of the mechanism of coagulation sedimentation process.
\end{abstract}

\section{Introduction}

At present, the main method of drinking water treatment is to promote the colloidal particles in the water destabilization and precipitation for purifing the water by adding coagulants to the original water. Coagulant dosage is important and affects the outlet water quality and the production cost.

In order to improve the drinking water quality, it is necessary to improve the performance of coagulant dosage control. However, because of the big time lag of coagulation sedimentation process, it is difficult to gather and adjust the control signals in time. Hence we can use a simulation model instead of the actual system, which can respond to the control signals quickly. If so, we can not only adjust control signals timely but also reduce the experiment cost.

It is difficult to model the coagulation sedimentation process due to the complexity. There has not been a unified model until now. Some scholars modeled the process based on experimental data, for example, $\mathrm{Na}$ et al. [1] obtained a transform function of coagulant dosage system, which is approximately a second-order inertia system including pure delay by data analysis. Besides, Guo et al. [2] assumed the process is made of multiple continuously stirred tank reactors in series and used population balance to model the process by calculating the flocculation rate according to the average particle size at different times. In this paper, we will model the process by analyzing the changes of the particle size distribution and calculating the removal rate of the particles of different sizes.

\section{Mechanism}

Coagulation sedimentation process is an important part of the drinking water treatment. It consists of mixing, flocculation and sedimentation. The water turbidity after sedimentation is important and we always control it by adjusting the coagulant dosage according to the original water quality in coagulant dosage control system. In this paper, we modeled the process through analyzing the effects of original water turbidity and coagulant dosage on the water turbidity after sedimentation.

\section{Mixing}

Assuming that coagulants mix with the original water through the pump and hydrolyze completely in the mixing basin. The mixing basin is a kind of continually stirred tank reactor with the following characteristics: the materials having different residence time in reactor mix up uniformly and momently; the concentration and composition of the materials in reactor are equal to that of the output materials of the reactor. Assuming that the effective volume of the basin is constant during the mixing process. From above, we can get Eq. (1) according to the population balances [2]. 


$$
V \frac{d x_{\text {out }}(t)}{d t}=q x_{\text {in }}(t)-q x_{\text {out }}(t)
$$

Then, give its Laplace transformation and the transform function of inlet and outlet coagulant concentration as follows:

$$
\begin{aligned}
\frac{x_{\text {out }}(s)}{x_{\text {in }}(s)} & =\frac{1}{(V / q) s+1} \\
\frac{y_{\text {out }}(s)}{y_{\text {in }}(s)} & =\frac{1}{(V / q) s+1}
\end{aligned}
$$

Where $x_{\text {in }}$ and $y_{\text {in }}$ are the colloid and coagulant concentration in the basin; $x_{\text {out }}$ and $y_{\text {out }}$ are the outlet colloid and coagulant concentration; $V$ is the effective volume of the basin; $q$ is the flow quantity.

When the $\mathrm{PH}$ value of the water is around 7, the hydrolysis products of coagulants are mainly positively charged aluminum hydroxide particles. If the aluminum hydroxide and the colloids in original water are uniform spheres with a diameter of $100 \mathrm{~nm}$ and $2 \mu \mathrm{m}$, and the surface charge concentration of them are same, we could think that single colloid would be neutralized and destabilized to adsorb 400 aluminum hydroxide particles [3]. But when coagulant dosage is more than the optimal dosage, the destabilized colloids would be restabilized adsorbing excess coagulant particles. So we can approximately calculate the number concentration of the destabilized colloids, $x_{d}$, as follows: when the dosage is less than the optimal dosage, $x_{d}$ is approximately equal to $y_{\text {out }} /\left(m_{y} \cdot 400\right)$; when the dosage is more than the optimal dosage, $x_{d}$ is approximately equal to $2 \cdot y_{\text {out }} /\left(m_{y} \cdot 400\right)-x_{\text {out }} / m_{x}$, where $m_{y}$ and $m_{x}$ are the quality of single coagulant and colloidal particle.

\section{Flocculation}

At present, most water plants use baffle or folded plate flocculation basin to deal with original water in China. The back mixing of the liquids flowing into the folded plate basin at different times are weak. So we assume that the folded plate flocculation basin is a tubular flow reactor. Based on the assumption, we can approximately analyze the size changes of the colloids in the water flowing into the flocculation basin at different times alone during the modeling process. Because the flocculation time is usually long, we can divide the flocculation process into $m$ segments. By calculating the flocculation rate in each segment, $r_{i}$, we can obtain the particle size distribution after flocculation.

Based on the flocculation model in size-continue form proposed by Smoluchowski in 1917, Hounslow et al. [5] discretized the full size distribution into geometric sections and proposed the rate of change of floc concentration, for the flocculation processes ignoring the fragmentation of flocs.

$$
\frac{d N_{i}}{d t}=r_{i}=N_{i-1} \sum_{j=1}^{i-2} 2^{j-i+1} \alpha_{i-1, j} \beta_{i-1, j} N_{j}+\frac{1}{2} \alpha_{i-1, i-1} \beta_{i-1, i-1} N_{i-1}^{2}-N_{i} \sum_{j=1}^{i-1} 2^{j-i} \alpha_{i, j} \beta_{i, j} N_{j}-N_{i} \sum_{j=i}^{\max } \alpha_{i, j} \beta_{i, j} N_{j}
$$

where $N_{i}$ is the number concentration of destabilized particles in section $i ; \alpha_{i, j}$ is the collision efficiency between the particles in section $i$ and $j ; \beta_{i, j}$ is the collision frequency.

Assume the collision efficiency of 1, i.e. $\alpha=1$. The collision frequency, $\beta_{i, j}$ is given by [6].

$$
\beta_{i, j}=\left(\frac{G}{6}\right) \cdot\left(d_{i}+d_{j}\right)^{3}
$$

Where $d_{i}$ and $d_{j}$ are the diameter of the particles in section $i$ and $j$; $G$ is the velocity gradient.

The volume of particles in section $i, V_{i}$, is $\left(b_{i}+b_{i-1}\right) / 2$, where $b_{i}$ and $b_{i-1}$ is upper and lower bounds of section $i$, and $b_{i}=2 b_{i-1}$ [7]. 
The destabilized colloids flocculated preliminarily during the mixing process. Assuming that the initial particle size distribution of the colloids in the flocculation basin, $N_{i, 0}$, is following [4].

$$
N_{i}=f\left(N_{0}, V_{i}\right)=\frac{N_{0}}{V_{0}}\left(\frac{V_{i}}{V_{0}}\right) \exp \left(\frac{-V_{i}}{V_{0}}\right)
$$

Where $N_{0}$ and $V_{0}$ are the initial total amount and average volume of the colloids.

\section{Sedimentation}

The water after flocculation flows into the sedimentation basin, and the flocs in the water sedimentate depending on the gravity. Assuming that the particles in the water distribute uniformly along the cross section when flowing into the basin; there is no interference between the particles, and the volume, shape and density of the particles do not change during the sedimentation; the velocity of the water anywhere in the basin are same and remain unchanged; the flocs sinking on the bottom of the basin are seemed removed and no longer return the water [4]. Based on the assumption above, the removal rate of the particles, $\eta$, is given by [4].

$$
\begin{aligned}
& \eta=\left(100-P_{0}\right)+\sum_{i=1}^{b} \frac{v_{i}}{v_{0}} d P \\
& v_{i}=\frac{1}{18} \cdot \frac{\Delta \rho}{\mu} \cdot g \cdot d_{i}^{2}
\end{aligned}
$$

Where $v_{0}$ is the capture velocity, i.e., the particles with a greater settling velocity than $v_{0}$ would be removed completely; $d P$ and $v_{i}$ are the percentage and settling velocity of the particles in section $i ; P_{0}$ and $b$ are the percentage and the biggest size section of the particles with a smaller velocity than $v_{0}$; $\Delta \rho$ is the density difference between the colloid and water; $\mu$ is the viscosity of the liquid.

\section{Model}

From above, we proposed a mechanism model for coagulation sedimentation process, and fig. 1 shows the flow chart.

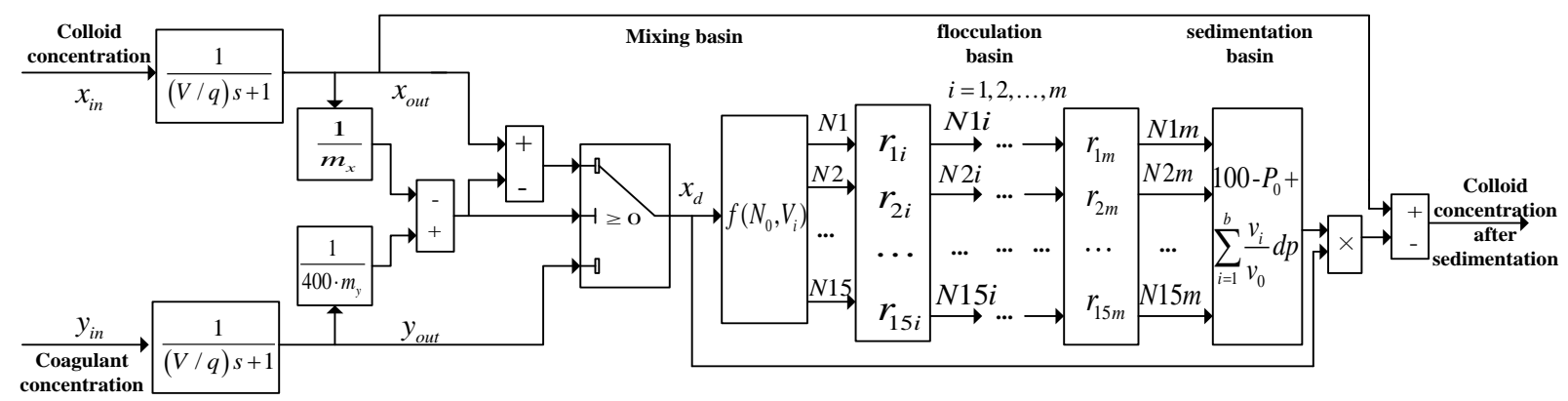

Fig. 1 the flow chart of the mechanism model of the coagulation sedimentation process

\section{Simulation Results and Analysis}

Assume parameters of the model as follows: the daily amount of water treatment is $100000 \mathrm{~m}^{3} / \mathrm{d}$; the effective volume of mixing basin is $6 \mathrm{~m}^{3}$; the average diameter of colloidal particles in the water after mixing is $10 \mu \mathrm{m}$; the $\mathrm{PH}$ value of the water is around 7; flocculation has two groups, and the flocculation time is $10 \mathrm{~min}$; The velocity gradient is $24.5 \mathrm{~s}^{-1}$; the diameter of the primary colloids and coagulant particles are $100 \mathrm{~nm}$ and $2 \mu \mathrm{m}$, and the density are $2.650 \mathrm{~g} / \mathrm{mL}$ and $2.420 \mathrm{~g} / \mathrm{mL}$; turbidities are translated from NTU to mg/L using the conversion, $2 \mathrm{mg} / \mathrm{L} * \mathrm{NTU}$ [8]; the flocculation process is divided into 60 segments, i.e. $\mathrm{m}=60$; the size of the particle is divided into 15 sections; the capture velocity is $2 \mathrm{e}-4 \mathrm{~m} / \mathrm{s}$; the viscosity of the liquid is $1.0 \mathrm{e}-4 \mathrm{~Pa} \cdot \mathrm{s}$.

Keep the original water turbidity 30NTU meanwhile adjusting the coagulant dosage to observe the changes of the water turbidity after sedimentation. The result is showed in fig. 2 . In order to study the 
dynamic relationship of original water turbidity and water turbidity after sedimentation, we increased the original water turbidity with different initial values suddenly as shown in fig. 3(a) and watched the changes of the water turbidity after sedimentation, while keeping the coagulant concentration $18 \mathrm{mg} / \mathrm{L}$. The water turbidity after sedimentation is showed in fig. 3(b).

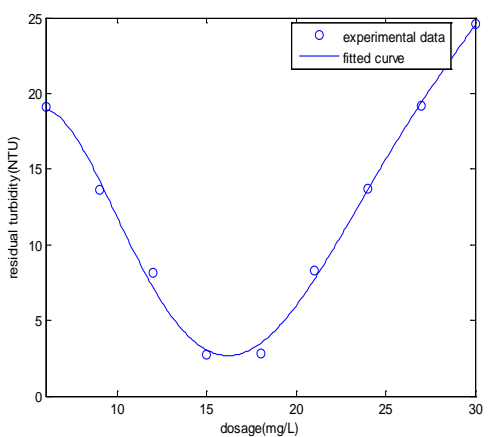

Fig. 2 water turbidity after sedimentation with different coagulant dosage

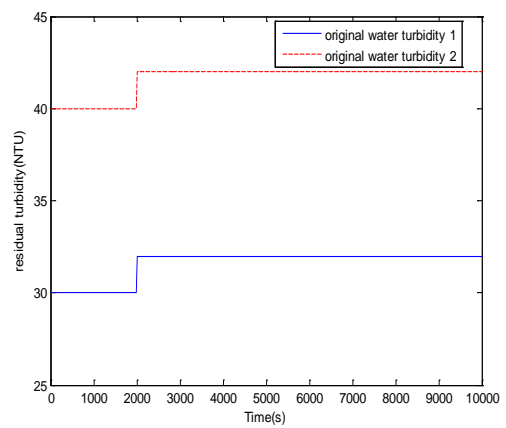

Fig.3(a) the original water turbidity

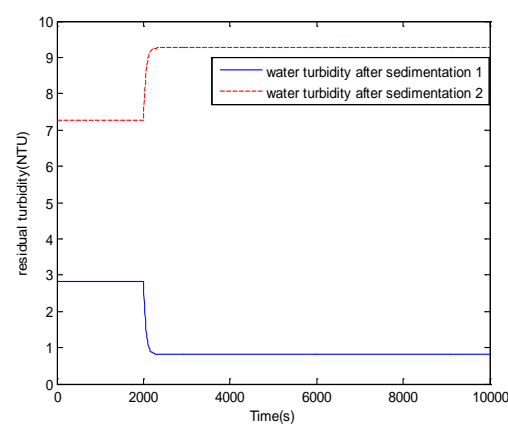

Fig.3(b) water turbidity after sedimentation

Fig. 2 shows that with the coagulant dosage increasing, the water turbidity after sedimentation first decreased and then increased. The main reason is that when the coagulant dosage is more than the optimal dosage, with the increase of the dosage, the destabilized colloids would restabilize to adsorb excess coagulants. Fig. 3(b) shows that when the initial value of the original water turbidity is different, the effect of original water turbidity on outlet water turbidity may be opposite, which also confirmed that the system is nonlinear.

\section{Conclusions}

A mechanism model was proposed in this paper. Based on the model, we simulated and analyzed the impact of original water turbidity and coagulant dosage on the water turbidity after sedimentation. The results matched with the practical analysis of the process. The mechanism model contributes to a further study on coagulation sedimentation process and provides advantages for studying coagulant dosage control. In addition, the model can be used to analyze the effect of each device parameter on water treatment, and has reference value for the construction of the water plants.

\section{Acknowledgments}

The work was supported by the National Natural Science Foundation of China(61174161,61304141, 61375077); the specialized Research Fund for the Doctoral Program of Higher Education of China (20130121130004); and the Fundamental Research Funds for the Central Universities in China (Xiamen University: No. CBX2014038).

\section{References}

[1] Jun Nan,Yanling Yang, Guibai Li: China water \& waste water Vol.19(2003), p:88-90, in chinese.

[2] Yufeng Guo, Jun Ma, Xuedong Zhang: Environmental Science and Information Application Technology, 2009. ESIAT 2009. International Conference on Vol.3, p: 288-291.

[3] Shuibo Xie, Yinghe Jiang in: Water Engineering. China Machine Press (2009), in chinese.

[4] Kumar S, Ramkrishna D: Chemical Engineering Science Vol. 51(8)(1996), p:1311-1332.

[5] M.J. Hounslow, R.L. Ryall, V.R: Marshall, AIChE J. 34 (1988) 1821.

[6] M. von Smoluchowski, Z. Phys. Chem. 92 (1917) 129.

[7] P.T. Spicer, S.E. Pratsinis, AIChE J. 42 (1996) 1612. 